\title{
Constitutive expression of small heat shock proteins in vegetative tissues of the resurrection plant Craterostigma plantagineum
}

\author{
Josefa Alamillo ${ }^{1,3, * *}$ Concepción Almoguera ${ }^{2, * *}$, Dorothea Bartels ${ }^{1}$ and Juan Jordano ${ }^{2, *}$ \\ ${ }^{1}$ Max-Planck-Institut für Züchtungforschung, Carl von Linné Weg 10, D-50829 Köln, Germany; \\ ${ }^{2}$ Instituto de Recursos Naturales y Agrobiología, C.S.I.C., Apartado 1052, 41080 Sevilla, Spain (*author \\ for correspondence); ${ }^{3}$ Present address: Departamento de Biotecnologia, Escuela Superior de Ingenieros \\ Agrónomos, 28040 Madrid, Spain (**J.A. and C.A. contributed equally to this work and are both considered \\ to be first author)
}

Received 11 May 1995; accepted in revised form 30 August 1995

Key words: Craterostigma, constitutive expression, desiccation tolerance, small heat-shock proteins, vegetative tissues

\begin{abstract}
Using antibodies raised against two sunflower small heat shock proteins (sHSPs), we have detected immunologically related proteins in unstressed vegetative tissues from the resurrection plant Craterostigma plantagineum. In whole plants, further accumulation of these polypeptides was induced by heat-shock or water-stress. In desiccation-intolerant Craterostigma callus tissue, we failed to detect sHSP-related polypeptides, but their expression, and the concurrent acquisition of desiccation tolerance was induced by exogenous abscisic acid (ABA) treatment. In untressed plants, the cross-reacting polypeptides were abundant in the roots and lower part of the shoots, where they showed homogeneous tissue-distributions. This constitutive expression is novel for vegetative tissues of higher plants, and resembles the expression patterns of sHSPs in desiccation-tolerant zygotic embryos and germinating seeds.
\end{abstract}

Angiosperms only tolerate severe protoplasmic dehydration during the late stages of zygotic embryogenesis in seed development. In some cases, the desiccation tolerance of the mature embryo is preserved during the early stages of germination. Later in development, the vegetative tissues of most plants become desiccation-intolerant. Exceptions to this comprise different species, which include mosses (i.e., Tortula ruralis), pteridophytes (Polypodium virginianum, Selaginella lepidophyla, etc.), and even a few higher plants termed resurrection plants [7, and references therein]. Among the latter, Craterostigma plantagineum
(Scrophulariaceae) has been used as model system for molecular analyses of desiccation tolerance. Most studies of Craterostigma have focused on the characterization of genes induced by dehydration [21, 22]. Many of these genes show sequence homologies with genes abundantly expressed in desiccation-tolerant zygotic embryos [reviewed in 3,4].

In the course of our studies on gene expression during late embryogenesis in sunflower, we isolated and characterized cDNAs encoding two seed-stored sHSPs, Ha HSP17.6 and $\mathrm{Ha}$ HSP17.9 [2, 11]. Their predicted amino acid se- 
quences matched that of members of two sHSP families classified as class I and class II cytosolic proteins [11]. In vegetative tissues, sHSPs have been shown to accumulate only in response to stress, and mostly under heat-shock conditions [24]. We showed that the sunflower seed-stored sHSPs accumulated during zygotic embryogenesis in the absence of thermal stress. Interestingly, the same polypeptides persisted during early germination, and a characteristic homogeneous and abundant tissue distribution of sunflower sHSPs was found to correlate with the desiccation tolerance of dry-seeds and young seedlings [11]. We also detected sHSP polypeptides in the vegetative tissues of sunflower, which were indistinguishable from those present in seeds. However, they only occurred in water-stressed plants, and showed a restricted tissue and organ distribution: the proteins were absent from the leaves, and were localized mostly in the lateral meristems of stressed stems and roots [1]. We and other groups have proposed the functional involvement of specific cytosolic sHSPs in the protection against and (or) recovery from the cellular damage caused by dehydration in the developing plant embryo [11, 13], and perhaps also in specialized vegetative tissues [1]. The present work addressed the question as to whether similar correlations between sHSP expression and desiccation tolerance persist in other plant systems used to study desiccation tolerance. We studied sHSP expression in the resurrection plant Craterostigma plantagineum grown under controlled environmental conditions. Our work presents the first evidence for the constitutive expression of plant sHSPs in vegetative tissues, and suggests a possible role for these proteins in the acquisition of desiccation tolerance in resurrection plants.

To investigate the expression of sHSPs in resurrection plants and its possible correlation with desiccation tolerance, we took advantage of a well studied model system, C. plantagineum. In this system, not only differentiated tissues are desiccation tolerant, but it is also possible to induce tolerance in undifferentiated callus tissue by pretreatment with ABA [5]. Controlled growth conditions were used to ensure that during our ex- periments Craterostigma plants and callus were not exposed to unwanted thermal or water stress. Protein extracts from different plant organs were analysed by SDS-PAGE, followed by immunodetection using two previously characterized antisera. These antisera contain antibodies that are specific, either for class I (i.e., anti-sunflower HSP17.6), or class II (i.e., anti sunflower HSP 17.9) plant cytosolic sHSPs [1]. Both antisera detected immunologically related polypeptides in the roots of unstressed plants. The antiHSP17.9 antiserum also detected homologous polypeptide(s) in control leaves, although here they were much less abundant (Fig. 1, lanes C). Desiccation treatment of whole plants led to a further moderate accumulation of these proteins. Thus, polypeptides, related to either HSP17.6 and HSP17.9, accumulated in desiccated roots, between 1.5- to 3-fold higher than the control levels. In leaves, desiccation only induced the accumulation of class II-related proteins (5-fold over control level). Rehydration of the desiccated plants resulted in a reduction of the accumulation levels for the polypeptides detected in roots, whereas in leaves this decrease would be slower (Fig. 1; compare lanes C, D and R). Similar results to those depicted in Fig. 1 were obtained using affinity-purified HSP17.6 and HSP17.9 antibodies (data not shown). Since these antibodies are very specific [1], as are other plant anti-sHSP antibodies [12, 13], our results strongly indicate that the detected polypeptides are class I and class II Craterostigma sHSPs. This is supported by the following considerations: the polypeptide(s) detected in unstressed $\mathrm{Cra}$ terostigma tissues by HSP17.9 and HSP17.6 antibodies migrated as a single band of $18.2 \mathrm{kDa}$ and as a doublet of 17.6 and $19.1 \mathrm{kDa}$, respectively (Fig. 1). These results are in agreement with the immuno-detection of similar size sHSP bands in both sunflower seeds [11], and in water- or heatstressed plants [1]. The Craterostigma proteins appeared to be only $1-2 \mathrm{kDa}$ smaller than sunflower sHSPs. In addition, heat-shock treatments of Craterostigma plants induced a further accumulation of apparently the same polypeptides in roots and in leaves. The abundance of the cross- 

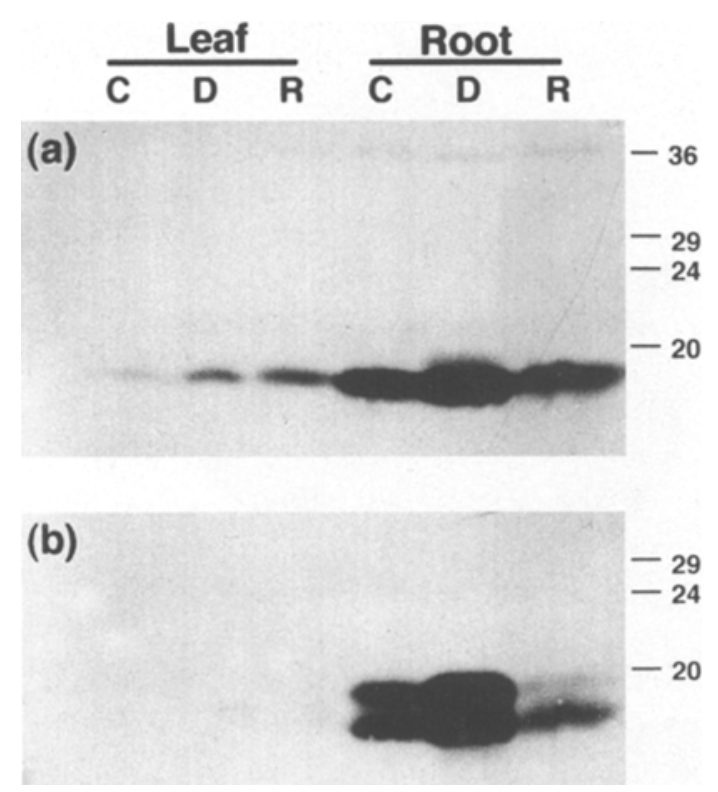

Fig. 1. Constitutive expression of anti-sHSP cross-reacting proteins in Craterostigma plantagineum Hoschst. (Scrophulariaceae). Proteins extracted from the vegetative organs of untreated (C), desiccated (D) or rehydrated plants (R) were separated by SDS-PAGE, transferred to nitrocellulose, and probed with HSP17.9 (panel a), and HSP17.6 (panel b) anti$\beta$-galactosidase-free antisera. In this experiment, the nitrocellulose membrane was first probed with the HSP17.6 antiserum, then antibody-stripped and re-probed with antiHSP17.9. Ordinate, molecular size in kDa. Plants were propagated under controlled-environment conditions: $60 \%$ relative humidity, day/night temperatures of $24^{\circ} \mathrm{C} / 20^{\circ} \mathrm{C}$, and a daily light period of $14 \mathrm{~h}$ [5]. Desiccation and dehydration experiments were performed in the same controlled environmental conditions: water was withheld from treated plants for two weeks. Desiccated plants were rehydrated by floating them in water for 15-20 h [5]. Plant samples were immediately frozen and ground in liquid nitrogen. Soluble proteins were extracted in Laemmli buffer [22]. Aliquots containing 30$60 \mu \mathrm{g}$ protein were separated in $12 \%$ polyacrylamide SDS gels and blotted onto nitrocellulose membranes using a semi-dry electrotransfer apparatus (Sartorius). Protein blots were processed for immuno-detection as described previously [1, 11]. The relative intensities of detected protein bands were measured by densitometric scanning of autoradiograms.

reacting polypeptides in heat-stressed organs was only about 3-fold higher than in unstressed organs (Fig. 2, and data not shown). Protein band(s) with approximately twice the apparent size $(35-38 \mathrm{kDa})$ of the sHSPs were also observed in samples from either desiccated or heat-
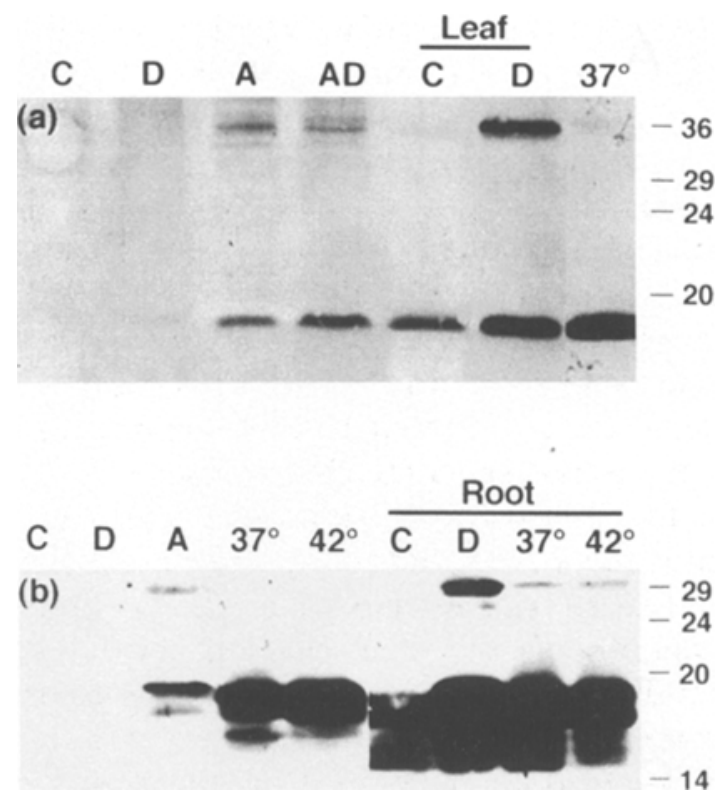

Fig. 2. Expression analysis in callus and comparisons with heat-shock-induced proteins. Protein extracts from untreated (C), desiccated (D), ABA-treated (A), and desiccated callus after ABA treatment (AD) were analysed as indicated in the legend to Fig. 1. Callus extracts following heat-shock treatments at $37^{\circ} \mathrm{C}$ or $42^{\circ} \mathrm{C}$ were also included (lanes $37^{\circ}$ and $42^{\circ}$ ). For comparisons, leaf or root samples from control and likewise treated plants, were simultaneously analysed with each antisera. a. Membrane probed with HSP17.9. b. membrane probed with HSP17.6. Callus was grown in vitro according to Bartels et al. [5] Callus was dried in a ventilating hood at an ambient temperature of $20-22^{\circ} \mathrm{C}$. For the abscisic acid (ABA, cis, trans, Sigma) treatment, the callus was incubated for four days on a medium containing $1 \mathrm{mM} \mathrm{ABA}$. For the heat-shock treatments, fully hydrated whole plants were placed in the dark for $2 \mathrm{~h}$ at either $37^{\circ} \mathrm{C}$ or $42^{\circ} \mathrm{C}$. Callus tissues were similarly heat-stressed but for $4 \mathrm{~h}$.

stressed plants (Fig. 2). These bands were only observed in samples with higher relative concentrations of sHSPs (compare Figs. 1 and 2, data not shown); and they might be explained by noncovalent protein-protein interactions that are not fully destroyed in SDS-PAGE, as has been reported for other plant sHSPs [9].

Similar immuno-detection experiments were performed with Craterostigma callus grown under identical environment conditions as the whole plants. Neither HSP17.6 nor HSP17.9 antisera recognized cross-reacting proteins in extracts from untreated callus. In contrast, heat-shock 
A

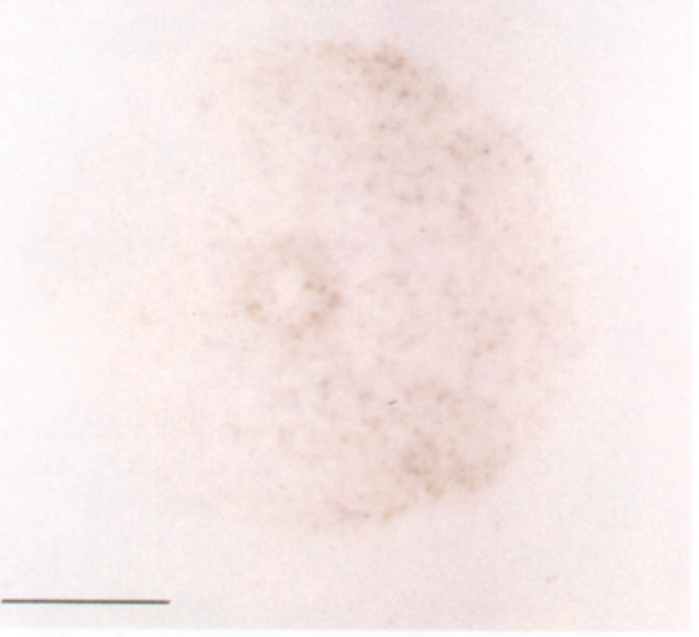

\section{C}
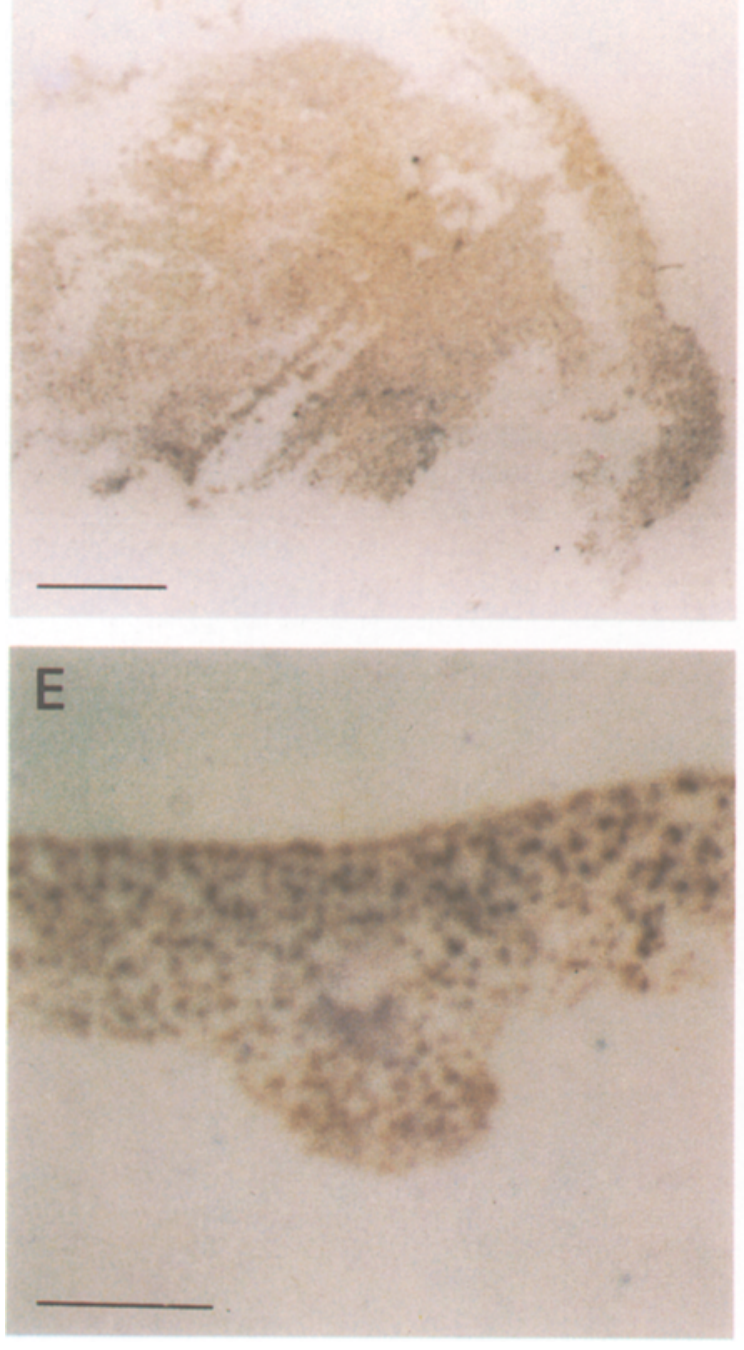

B
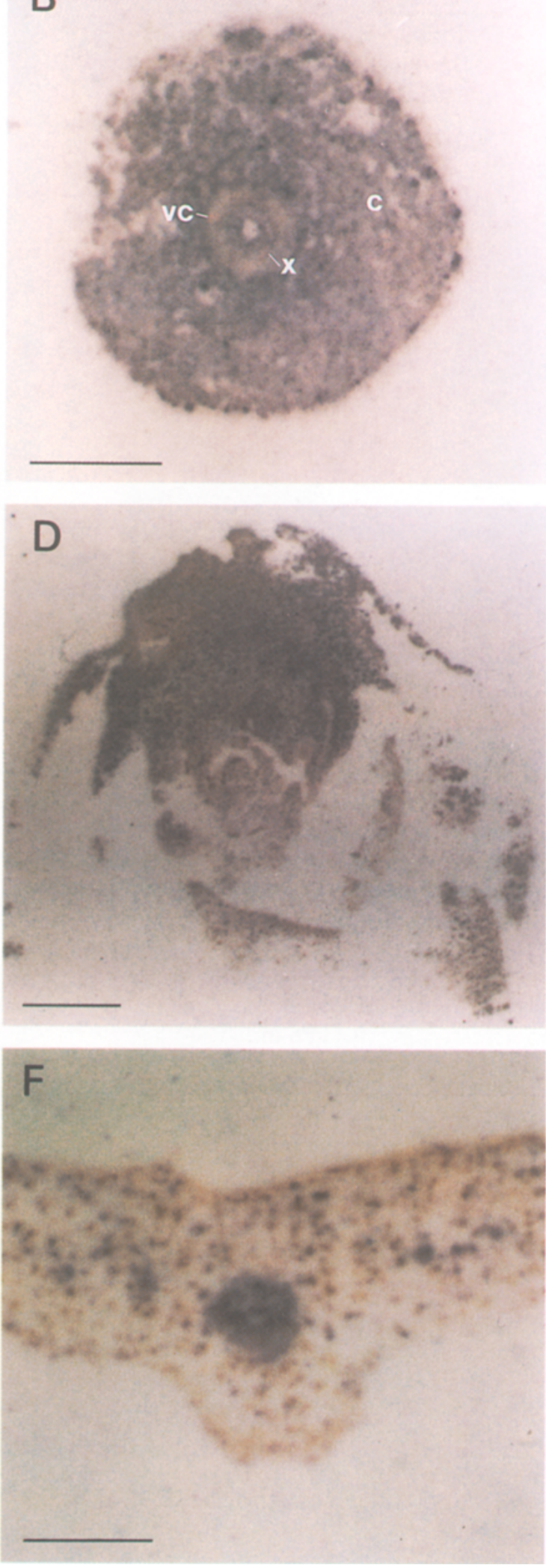
treatment of callus, at either $37^{\circ} \mathrm{C}$ or $42^{\circ} \mathrm{C}$, resulted in the accumulation of sHSPs with the same electrophoretical mobility as the polypeptides constitutively present in the leaves and roots of unstressed plants (Fig. 2, compare lanes C, $37^{\circ} \mathrm{C}$ and $42^{\circ} \mathrm{C}$ ). This provides further evidence for the immunological identification of these polypeptides as either class I or class II, cytosolic Craterostigma sHSPs. In addition, the absence of sHSPs from untreated callus tissue confirms that the constitutive expression of sHSPs in the vegetative tissues of whole plants was not a consequence of uncontrolled heat-stress under our experimental conditions. Another interesting observation was made using the callus system: ABA treatment of callus induced the accumulation of both class I and class II sHSPs at normal growth temperature, but the desiccation of callus did not induce these polypeptides. The ABAinduced polypeptides were indistinguishable from the proteins constitutively present in the vegetative tissues of unstressed plants (Fig. 2). Since Craterostigma roots and leaves are desiccationtolerant, whereas callus acquires desiccation tolerance only after ABA treatment [5], and the expression of sHSPs at normal growth temperature correlates, in both cases, with the desiccation tolerant phenotype, normal growth temperature sHSP expression might be used as a molecular marker for desiccation tolerance.

The detection of sHSPs in the unstressed vegetative tissues of Craterostigma, is a novel observation in plants. The current notion is that, in vegetative tissues, sHSPs are only synthesized in response to heat and other stresses [24], including water-stress [1]. Some evidence has suggested that there could be exceptions to this notion. For example, an Arabidopsis thaliana cDNA clone encoding a $17.6 \mathrm{kDa}$ protein, with $64-68 \%$ amino acid identity to class II sHSPs, was cloned from leaf mRNA of plants grown at $21^{\circ} \mathrm{C}$ [6]. An- other sHSP cDNA was cloned from mRNA of unstressed, seven-day-old, Papaver somniferum seedlings [14]. In addition, sHSP mRNAs accumulated during plant gametogenesis and somatic embryogenesis at normal growth temperatures [reviewed in 15]; but it is not clear whether sHSP mRNAs are translated in all these cases. Recent work has demonstrated transcriptional activation of specific sHSP promoters in the flower tissues of unstressed transgenic plants [23], and during pollen embryogenesis [25]. However, in the absence of thermal stress, plant sHSPs have been shown to be translated only during seed maturation [11, 13; and references therein]. In seeds, the temporal $[11,13]$ as well as the spatial [11] expression patterns of cytosolic sHSPs have been shown to be similar to those of Lea (late embryogenesis-abundant) proteins. This has led to the hypothesis that specific sHSPs are involved in desiccation tolerance $[11,13]$. The homogeneous and abundant tissue-distribution of sunflower sHSPs was found to correlate with the desiccation tolerance of dry-seeds and young seedlings [11]. For that reason, the investigation of the distribution of sHSPs in unstressed vegetative organs of Craterostigma is of particular interest. A summary of the results from our sHSP tissue-print localization experiments is presented in Fig. 3. In the roots and lower part of the shoots, polypeptides, immunologically related to either class I or class II sHSPs, showed both identical localization (Fig. 3B, 3D and data not shown) and abundance to that in the developing seeds of sunflower (the latter deduced from comparable immunoreaction times, data not shown). The distribution of these polypeptides was homogenous and it extended to the cortex parenchyma. In contrast, and as expected from results shown in Fig. 1, only HSP17.9-related polypeptides were detected in leaves, and were not only much less abundant than in the roots but also mostly local-

Fig. 3. Tissue-print immuno-localizations. Prints of cross-sections from unstressed plants: through the main root (A, B); immediately above the basal rosette ('lower parts of shoot': C, D); and through the leaf (E, F). Probed with HSP 17.9 (B, F) or HSP17.6 (D) anti- $\beta$-galactosidase-free antisera. $\mathrm{A}, \mathrm{C}$, and $\mathrm{E}$ probed with the corresponding pre-immune antiserum. Abbreviations: $\mathrm{c}$, root cortex; vc, vascular cylinder; p, phloem; $x$, xylem. Scale bars $=0.5 \mathrm{~mm}(\mathrm{~A}, \mathrm{~B}, \mathrm{E}, \mathrm{F})$, or $1.0 \mathrm{~mm}(\mathrm{C}, \mathrm{D})$. Tissue prints were processed for immuno-detection following the procedures and precautions described in Almoguera et al. [1]. 
ized in the tissues of primary and secondary vascular bundles (Fig. 3F). These expression patterns are different from the ones previously described in sunflower vegetative tissues, where sHSPs are only observed in response to water stress and in the lateral meristems of stems and roots, but not in leaves [1]. The distribution of sHSPs in Craterostigma rather resembles the homogeneous localization of sHSPs observed in sunflower seeds and young seedlings [11]. In the resurrection plant, the homogeneous sHSP expression pattern and its correlation with desiccation tolerance are maintained beyond germination, at least in the root and the lower parts of the shoot. The more restricted sHSP expression pattern in leaves indicates, however, that gene products other than sHSPs are required for the desiccation tolerance of the whole plant. This is confirmed by the failure to confer water-stress tolerance by constitutive over-expression of seedstored sunflower sHSPs in transgenic tobacco (Almoguera et al., unpublished observations). Although the expression of sHSPs is not sufficient to confer desiccation tolerance, it could still contribute to the ability to withstand cytosolic dehydration: for example, by helping to protect and/or repair damaged sub-cellular components in specific tissues. The molecular chaperon activities recently demonstrated in vitro for animal and plant sHSPs $[17,18]$ could be important for such roles.

Craterostigma genes, thought to be relevant for desiccation tolerance, are induced in callus in response to exogenous ABA. Most of these genes are expressed in vegetative tissues in response to desiccation [3]. The present work suggests that constitutive sHSPs could also be a component of desiccation tolerance in resurrection plants. The potential functional significance of constitutive gene expression related to dehydration survival has been indicated, for example, by the detection of proteins immunologically related to dehydrins in the fully hydrated desiccation-tolerant moss Tortula ruralis [8]. Dehydrins are proteins thought to be involved in water-stress tolerance, which are also expressed during late zygotic embryogenesis [10]. Likewise, in Craterostigma, the promoter of dehydrin homologous gene CDeT6-19 has been shown to be active in unstressed vegetative tissues [19]; and the protein encoded by CDeT6-19 (dsp16) was present at low levels in unstressed plants [22]. However, neither over-expression of a single dehydrin-like protein in tobacco, nor of other water-stress inducible Craterostigma proteins, was sufficient to confer phenotypical changes related to desiccation tolerance [16]. Thus, desiccation tolerance in resurrection plants would be a consequence of the concerted action of an array of different genes that results in changes in protein synthesis and carbohydrate metabolism [3]. Work reported here brings sHSPs genes into this complex scheme. Constitutive genes, including those encoding cytosolic sHSPs, could complement the roles of other desiccation-induced genes by providing some protection even in the fully hydrated state. $\mathrm{Cra}$ terostigma gene promoter analysis has suggested that there might be differences in gene regulation between desiccation-tolerant and non-tolerant plants: In transgenic tobacco, the Craterostigma promoter cDeT27-45 was only active in desiccation-tolerant seeds and pollen grains, but not in other water-stressed non-tolerant tissues; whereas, in Craterostigma, the cDeT27-45 protein was strongly induced in all organs by desiccation $[20,22]$. The expression of sHSPs well beyond embryogenesis and germination in the unstressed tissues of Craterostigma indicates another of these differences in the regulation of gene expression, although its actual functional significance remains to be determined.

\section{Acknowledgements}

We would like to thank Dr José Manuel Pardo for his critical reading of the manuscript. J.A. was supported by a Human Capital Mobility EU fellowship. CA was a recipient of a post-doctoral ('reincorporación') contract from Spanish CICYT. Collaboration between the Cologne and Seville groups was inspired in meetings organized under the 'Interdrought' EU concerted action (AIR3-CT92-0742). Part of this work was funded through grant BIO93-0023, awarded to J.J. 


\section{References}

1. Almoguera C, Coca MA, Jordano J: Tissue-specific expression of sunflower heat shock proteins in response to water stress. Plant J 4: 947-958 (1993).

2. Almoguera $\mathrm{C}$, Jordano J: Developmental and environmental concurrent expression of sunflower dry-seedstored low molecular-weight heat-shock protein and Lea mRNAs. Plant Mol Biol 19: 781-792 (1992).

3. Bartels D, Alexander R, Schneider K, Elster R, Velasco R, Alamillo J, Bianchi G, Nelson D, Salamini F: Desiccation-related gene products analyzed in a resurrection plant and in barley embryos. In: Close TJ, Bray EA (eds), Plant Responses to Cellular Dehydration During Environmental Stress. Current Topics of Plant Physiology vol. 10, pp. 119-127 (1993).

4. Bartels D, Nelson D: Approaches to improve stress tolerance using molecular genetics. Plant Cell Environ 17: 659-667 (1994).

5. Bartels D, Schneider K, Terstappen G, Piatkowski D, Salamini F: Molecular cloning of abscisic acid-modulated genes which are induced during desiccation of the resurrection plant Craterostigma plantagineum. Planta 181: 27-34 (1990).

6. Bartling D, Bütler H, Liebeton K, Weiler EW: An Arabidopsis thaliana $\mathrm{cDNA}$ clone encoding a $17.6 \mathrm{kDa}$ class II heat shock protein. Plant Mol Biol 18: 1007-1008 (1992).

7. Bewley JD: Physiological aspects of desiccation tolerance. Annu Rev Plant Physiol 30: 195-238 (1979).

8. Bewley JD, Reynolds TL, Oliver MJ: Evolving strategies in the adaptation to desiccation. In: Close TJ, Bray EA (eds) Plant Responses to Cellular Dehydration During Environmental Stress. Current Topics of Plant Physiology vol. 10, pp. 193-201 (1993).

9. Chen Q, Osteryoung K, Vierling E: A 21-kDa chloroplast heat shock protein assembles into high molecular weight complexes in vivo and in organelle. J Biol Chem 269: 13216-13223 (1994).

10. Close TJ, Fenton RD, Yang A, Asghar R, DeMason DA, Crone DE, Meyer NC, Moonan F: Dehydrin: The protein. In: Close TJ, Bray EA (eds) Plant Responses to Cellular Dehydration During Environmental Stress. Current Topics of Plant Physiology vol. 10, pp. 104-118 (1993).

11. Coca MA, Almoguera C, Jordano J: Expression of sunflower low-molecular-weight heat-shock proteins during embryogenesis and persistence after germination: localization and possible functional implications. Plant $\mathrm{Mol}$ Biol 25: 479-492 (1994).

12. DeRocher AE, Helm KW, Lauzon LM, Vierling E: Expression of a conserved family of cytoplasmic low molecular weight heat shock proteins during heat stress and recovery. Plant Physiol 96: 1038-1047 (1991).
13. DeRocher AE, Vierling E: Developmental control of small heat shock protein expression during pea seed maturation. Plant J 5: 93-102 (1994).

14. Facchini PJ, De Luca V: A cDNA encoding a low molecular mass heat-shock protein from opium poppy. Plant Physiol 106: 811-812 (1994).

15. Heikkila JJ: Heat shock gene expression and development. I. An overview of fungal, plant and poikilothermic animal developmental systems. Devel Genet 14: 1-5 (1993).

16. Iturriaga G, Schneider K, Salamini F, Bartels D: Expression of desiccation-related proteins from the resurrection plant Craterostigma plantagineum in transgenic tobacco. Plant Mol Biol 20: 555-558 (1992).

17. Jakob U, Gaestel M, Engel K, Buchner J: Small heat shock proteins are mólecular chaperones. J Biol Chem 268: $1517-1520$ (1993).

18. Lee GJ, Pokala N, Vierling E: Structure and in vitro molecular chaperone activity of cytosolic small heat shock proteins from pea. J Biol Chem, 270: 10432-10438 (1995).

19. Michel D, Furini A, Salamini F, Bartels D: Structure and regulation of an ABA- and desiccation responsive gene from the resurrection plant Craterostigma plantagineum. Plant Mol Biol 24: 549-560 (1994).

20. Michel D, Salamini F, Bartels D, Dale P, Baga M, Szalay A: Analysis of a desiccation and ABA-responsive promoter isolated from the resurrection plant Craterostigma plantagineum. Plant J 4: 29-40 (1993).

21. Piatkowski D, Schneider K, Salamini F, Bartels D: Characterization of five abscisic acid-responsive cDNA clones isolated from the desiccation-tolerant plant Craterostigma plantagineum and their relationship to other water-stress genes. Plant Physiol 94: 1682-1688 (1990).

22. Schneider K, Wells B, Schmelzer E, Salamini F, Bartels D: Desiccation leads to the rapid accumulation of both cytosolic and chloroplastic proteins in the resurrection plant Craterostigma plantagineum Hochst. Planta 189: 120-131 (1993).

23. Tsukaya $H$, Takahashi $T$, Naito $S$, Komeda $Y$ : Floral organ-specific and constitutive expression of an Arabidopsis thaliana heat-shock HSP18.2::GUS fusion gene is retained even after homeotic conversion of flowers by mutation. Mol Gen Genet 237: 26-32 (1993).

24. Vierling E: The roles of heat shock proteins in plants. Annu Rev Plant Physiol Plant Mol Biol 42: 579-620 (1991).

25. Zarsky V, Garrido D, Eller N, Tupy J, Vicente O, Schöffl F, Heberle-Bors E: The expression of a small heat shock gene is activated during induction of tobacco pollen embryogenesis by starvation. Plant Cell Environ 18: 139147 (1995). 